\title{
New Inhibitors of Indoleamine 2,3-Dioxygenase 1: Molecular Modelling Studies, Synthesis and Biological Evaluation
}

Antonio Coluccia, ${ }^{a}$ Sara Passacantilli, ${ }^{a}$ Valeria Famiglini, ${ }^{a}$ Manuela Sabatino, ${ }^{b}$ Alexandros Patsilinakos, ${ }^{\text {be }}$ Rino Ragno, ${ }^{\text {be }}$ Carmela Mazzoccoli, ${ }^{c}$ Lorenza Sisinni, ${ }^{c}$ Alato Okuno, ${ }^{\mathrm{d}}$ Osamu Takikawa ${ }^{\mathrm{d}}$ Romano Silvestri ${ }^{\mathrm{a}}$ and Giuseppe La Regina ${ }^{\mathrm{a}, *}$

astituto Pasteur - Fondazione Cenci Bolognetti, Dipartimento di Chimica e Tecnologie del Farmaco, Sapienza Università di Roma, Piazzale Aldo Moro 5, I-00185 Rome, Italy ${ }^{b}$ Rome Center for Molecular Design, Dipartimento di Chimica e Tecnologie del Farmaco, Sapienza Università di Roma, Piazzale Aldo Moro 5, I-00185 Rome, Italy ${ }^{c}$ Laboratorio di Ricerca Pre-Clinica e Traslazionale, Istituto di Ricovero e Cura a Carattere Scientifico (IRCCS), Centro di Riferimento Oncologico della Basilicata, Via Padre Pio 1, I-85028 Rionero in Vulture, Italy

${ }^{\mathrm{d} N a t i o n a l ~ I n s t i t u t e ~ f o r ~ L o n g e v i t y ~ S c i e n c e s, ~ N a t i o n a l ~ C e n t e r ~ f o r ~ G e r i a t r i c s ~ a n d ~}$ Gerontology 35 Gengo, Morioka, Obu, Aichi 474-8511, Japan

${ }^{\mathrm{e} A l c h e m i c a l ~ D y n a m i c s ~ s . r .1 ., ~ P i a z z a l e ~ A l d o ~ M o r o ~ 5, ~ I-00185 ~ R o m e, ~ I t a l y ~}$

*To whom correspondence should be addressed. E-mail: giuseppe.laregina@uniroma1.it. Phone:+39 0649913404. Fax: +39 0649913993.

\section{Supporting Information}

\section{Contents}

${ }^{13}$ C NMR and MS data. Compounds 7, 9, 13, 19, 22 and 25

Figure 1S. Snapshot of IDO1/L-Trp complex trajectory

Figure 2S. Effect of compound $\mathbf{5}$ on IDO1 activity

Figure 3S. Effect of compound $\mathbf{5}$ on TDO2 activity

Figure 4S. Inhibition of IDO1 activity by compound 5

Figure 5S. Average activity contribution plots (C and HD probes)

Figure 6S. C probe average activity contribution plots (compounds 12 and 30)

Figure 7S. C probe average activity contribution plots (compounds 28 and 30)

Figure 8S. C probe average activity contribution plots (compound 12)

Figure 9S. Average activity contribution plots (C and HD probes)

Table 1S. 3-D QSAR results with the VPO, simulated annealing and Y-scrambling

Table 2S. 2-D and 3-D structures, features and their values of the selected template molecules

Table 3S. Grid optimization and VPO analysis parameters

Table 4S. AdmetSAR data for diverse chemicals associated with known absorption, distribution, metabolism, excretion and toxicity profiles for compounds $\mathbf{5}$ and $\mathbf{2 1}$

Table 5S. Calculated drug likeness properties for compounds 5 and 21 by Molinspiration web server

Table 6S. Volsurf calculated ADMET properties for compounds 5 and 21 


\section{${ }^{13} \mathrm{C}$ NMR and MS data}

(5-Chloro-1H-indol-3-yl)(2-methoxyphenyl)methanone (7). ${ }^{13} \mathrm{C}$ NMR (DMSO-d 6 ): $\delta$ 55.91, 112.39, 114.45, 116.88, 120.67, 120.74, 123.46, 127.14, 128.66, 131.06, 131.30, 135.75, 137.88, 156.54, 189.92 ppm. MS (ESI) calcd for $\mathrm{C}_{16} \mathrm{H}_{12} \mathrm{ClNO}_{2}$ : 285.1; found $(\mathrm{M}+\mathrm{H})^{+}: 286.1$.

5-Chloro-3-(phenylthio)-1H-indole (9). ${ }^{13} \mathrm{C}$ NMR (DMSO-d6): $\delta$ 99.83, 114.53, 117.74, 122.68, 125.41, 125.47, 125.94, 129.38, 130.42, 134.67, 135.69, 139.08 ppm. MS (ESI) calcd for $\mathrm{C}_{14} \mathrm{H}_{10} \mathrm{ClNS}: 259.0$; found $(\mathrm{M}+\mathrm{H})^{+}: 260.0$.

5-Chloro-3-(o-tolylthio)-1H-indole (13). ${ }^{13} \mathrm{C}$ NMR (DMSO-d 6 ): $\delta$ 19.84, 99.20, 114.55, $117.74,122.67,125.11,125.39,126.85,130.37,130.50,134.23,134.76,135.76$, 138.04 ppm. MS (ESI) calcd for $\mathrm{C}_{15} \mathrm{H}_{12} \mathrm{CINS}: 273.0$; found $(\mathrm{M}+\mathrm{H})^{+}: 274.0$.

5-Ethoxy-3-((2-methoxyphenyl)thio)-1H-indole (19). ${ }^{13} \mathrm{C}$ NMR (DMSO-d6): $\delta 15.19$, $56.12,63.79,98.00,101.21,111.04,113.17,113.59,121.29,125.42,125.75,128.11$, 130.17, 132.20, 133.42, 153.87, 155.15 ppm. MS (ESI) calcd for $\mathrm{C}_{17} \mathrm{H}_{17} \mathrm{NO}_{2} \mathrm{~S}: 299.1$; found $(\mathrm{M}+\mathrm{H})^{+}: 300.1$.

5-Chloro-3-((3,5-dimethoxyphenyl)thio)-2-methyl-1H-indole (22). ${ }^{13} \mathrm{C}$ NMR (DMSO$\left.d_{6}\right): \delta 12.15,55.53,96.56,97.08,103.58,113.45,117.09,121.84,125.18,131.46$, $134.55,141.39,144.79,161.23$ ppm. MS (ESI) calcd for $\mathrm{C}_{17} \mathrm{H}_{16} \mathrm{ClNO}_{2} \mathrm{~S}: 333.1$; found $(\mathrm{M}+\mathrm{H})^{+}: 334.1$.

3-((3,5-Dimethoxyphenyl)thio)-1H-indole (25). ${ }^{13} \mathrm{C}$ NMR (DMSO-d $)$ ): $\delta 55.52,97.10$, 99.53, 103.97, 112.79, 118.78, 120.58, 122.58, 129.14, 132.97, 137.12, 142.06, 161.12 ppm. MS (ESI) calcd for $\mathrm{C}_{16} \mathrm{H}_{15} \mathrm{NO}_{2} \mathrm{~S}: 285.1$; found $(\mathrm{M}+\mathrm{H})^{+}: 286.1$. 


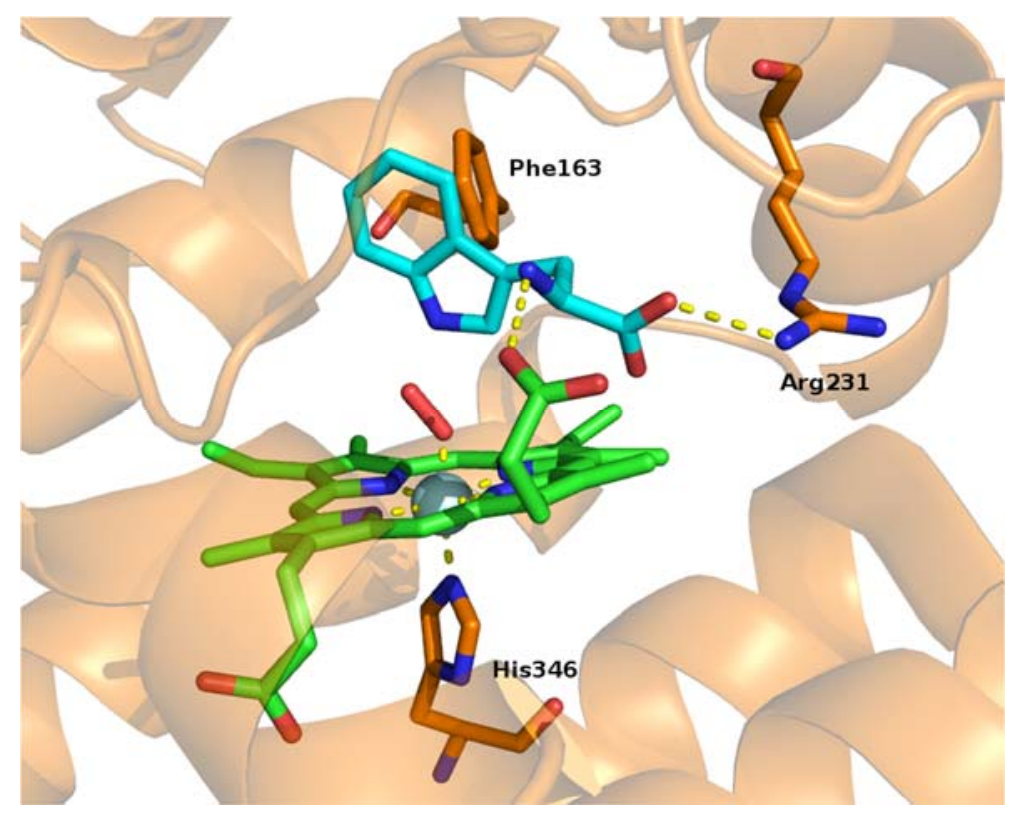

Figure 1S. Snapshot of IDO1/L-Trp complex trajectory. L-Trp is reported as cyan stick, heme as green stick, iron as silver sphere and oxygen molecule as red stick. The IDO structure was showed as cartoon. H-bonds are depicted as yellow dot lines.

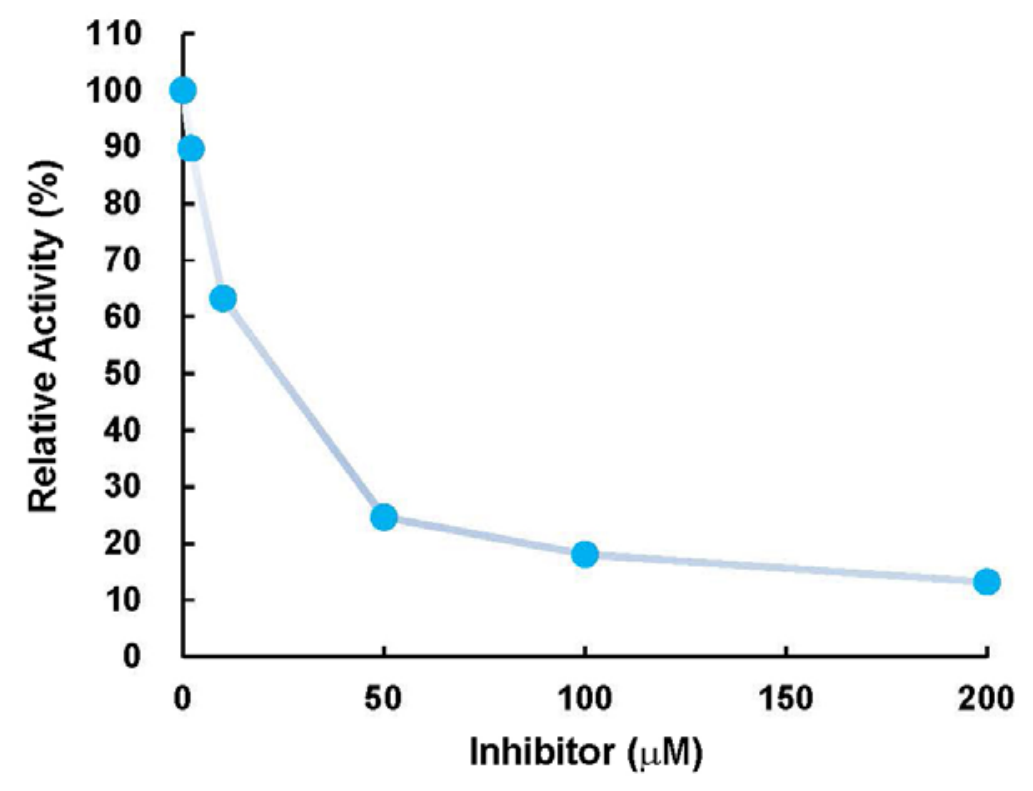

Figure 2S. Inhibition of IDO1 activity by compound $\mathbf{5}$. 


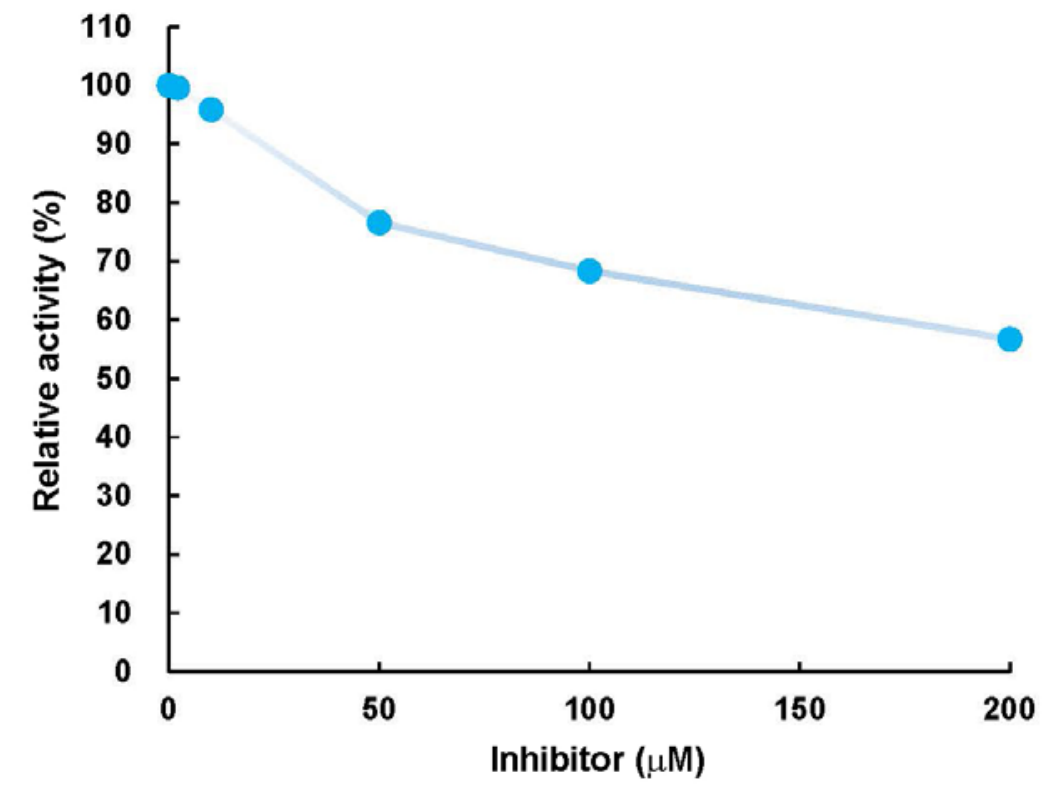

Figure 3S. Inhibition of TDO2 activity by compound 5 .

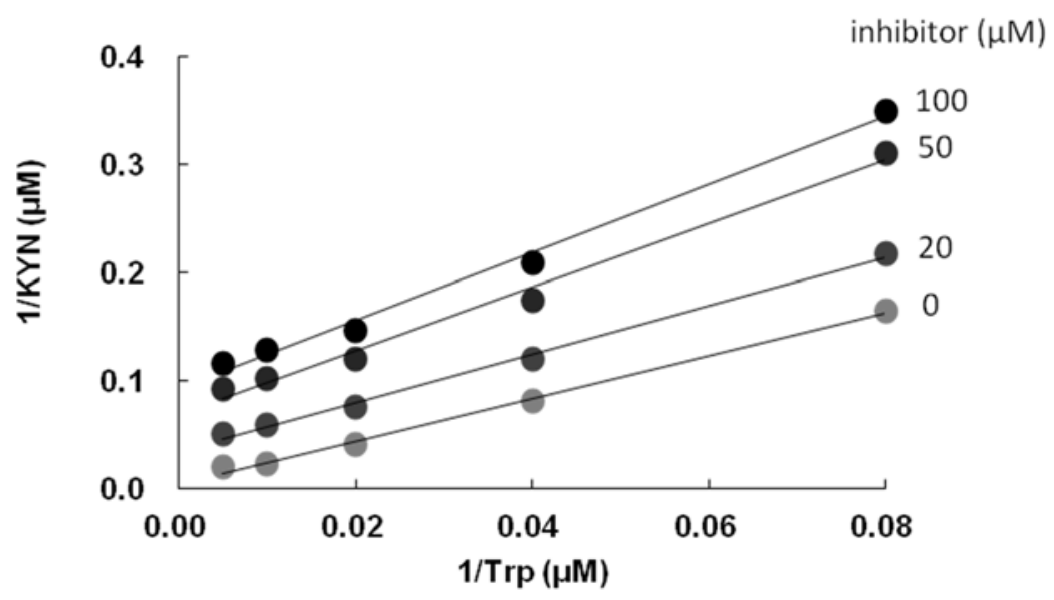

Figure 4S. Inhibition of IDO1 activity by compound 5. Kynurenine (KYN) concentration versus L-Trp (Trp) for the reaction catalyzed by IDO1 with different concentration of compound $\mathbf{5}$. 


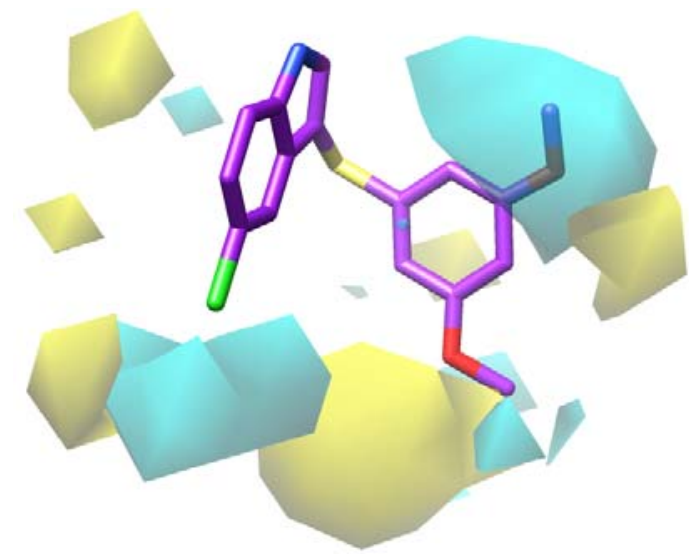

$\mathbf{A}$

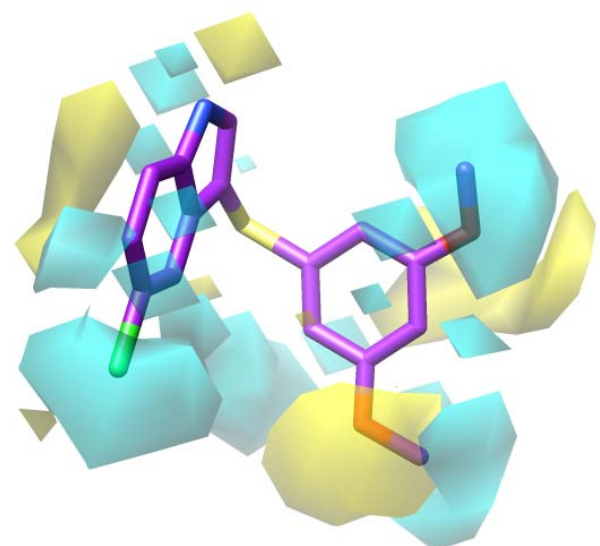

B

Figure 5S. Average activity contribution plots (A: probe C; B: probe HD). The most active derivative $\mathbf{2 1}$ is also shown for interpretation.

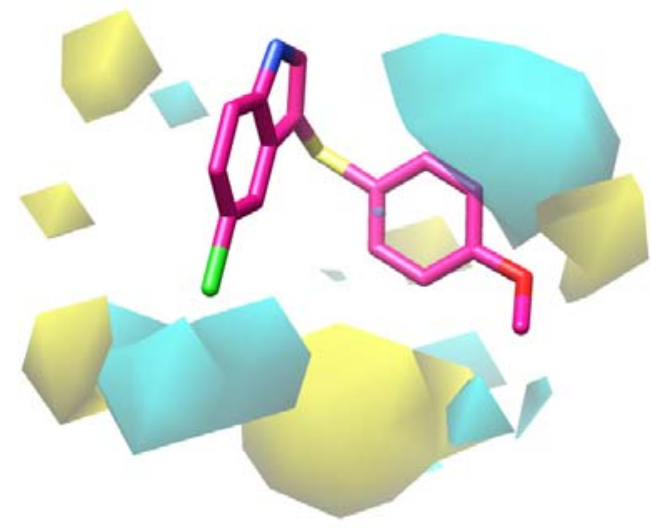

A

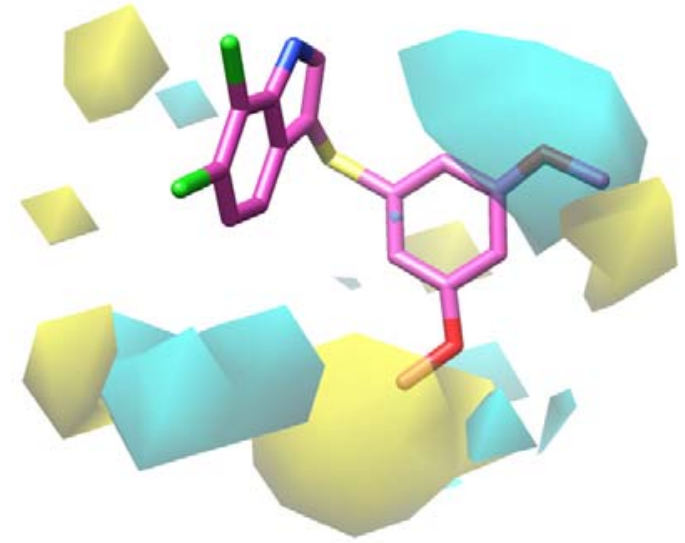

B

Figure 6S. $\mathrm{C}$ probe average activity contribution plots (A: compound 12; B: compound 30). 


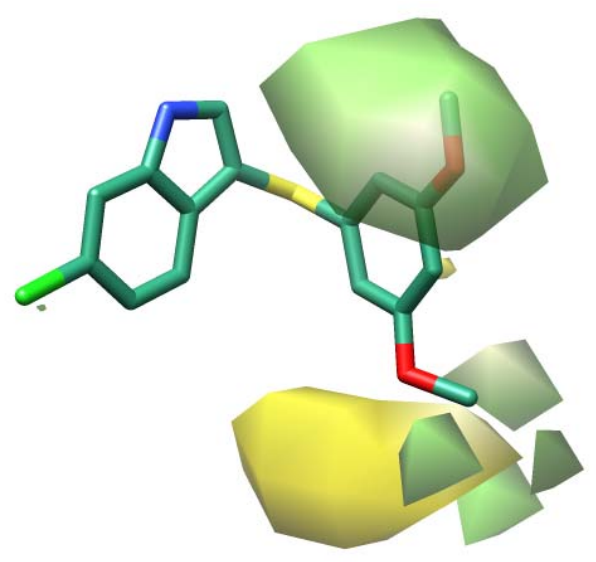

A

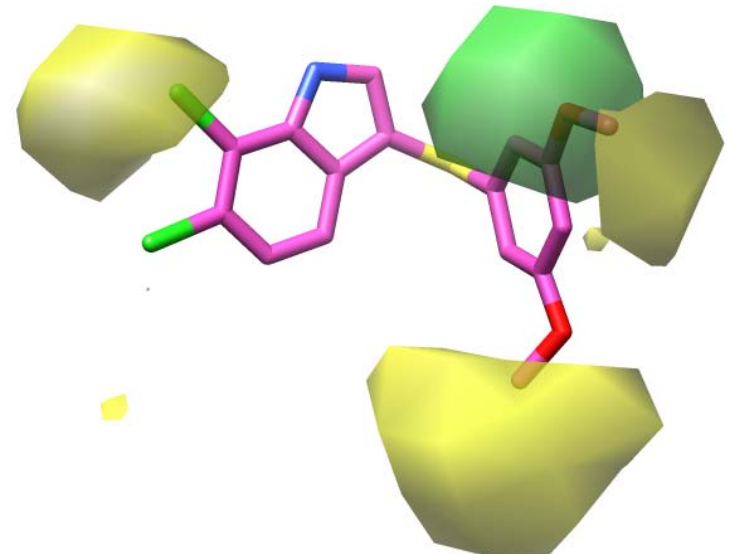

B

Figure 7S. C probe average activity contribution plots (A: compound 28; B: compound 30). Green and yellow positive and negative contributes, respectively.

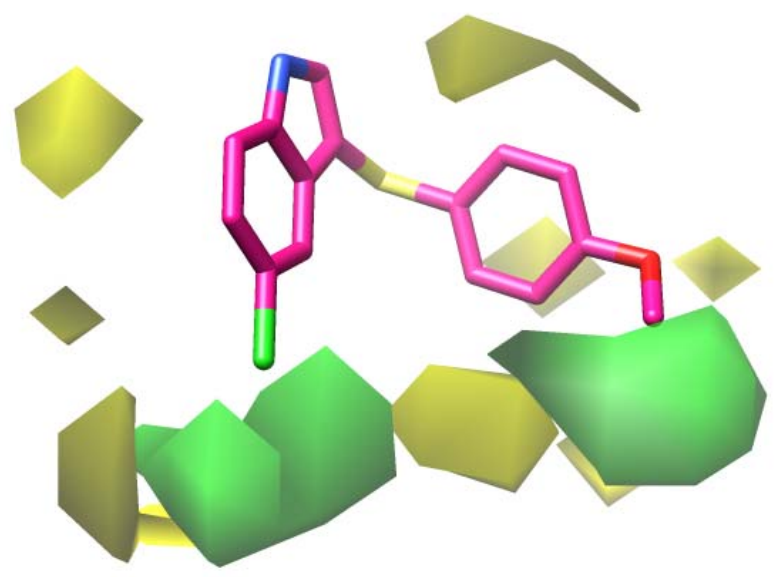

Figure 8S. C probe average activity contribution plots (compound 12). Green and yellow positive and negative contributes, respectively. 


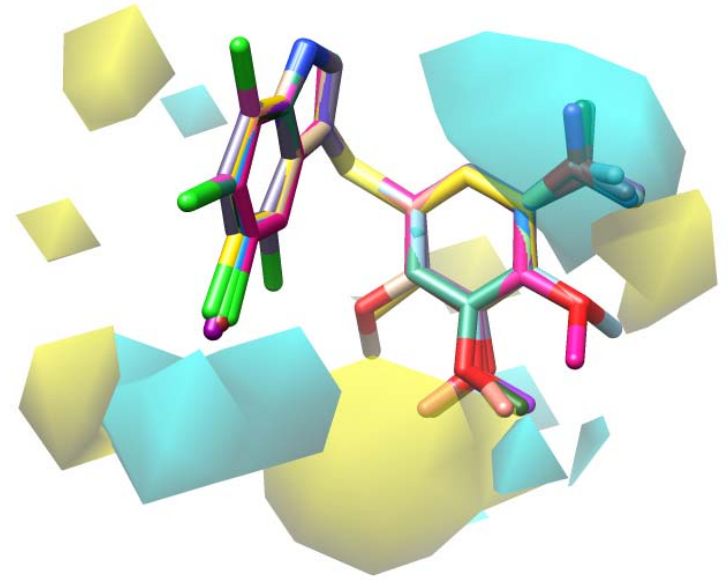

A

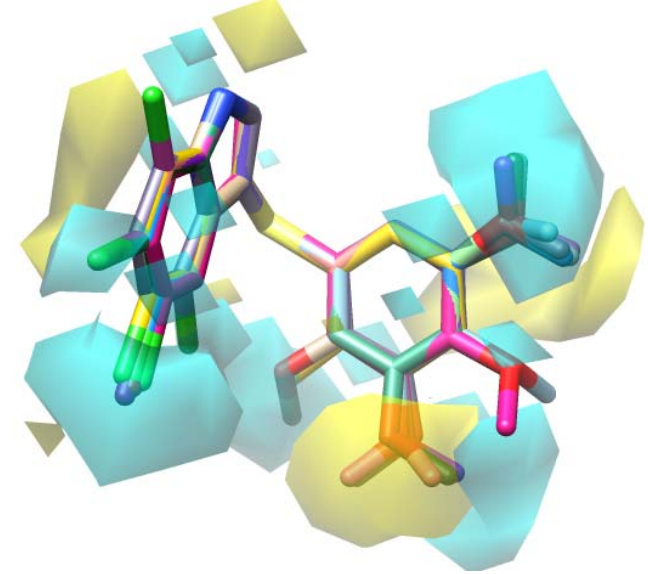

B

Figure 9S. Average activity contribution plots (A: C probe; $\mathbf{B}$ : HD probe). 
Table 1S. 3-D QSAR Results with the VPO, Simulated Annealing and Yscrambling

\begin{tabular}{cccccccccccc}
\hline Probe & $\begin{array}{c}\mathbf{G r i d} \\
\text { spacing }^{a}\end{array}$ & Cutoff $^{b}$ & Zeroing $^{c}$ & MinStd $^{d}$ & $\boldsymbol{r}^{2 e}$ & $\boldsymbol{q}^{2 f}$ & $\mathbf{S D E P}^{g}$ & $\boldsymbol{q}_{\mathbf{Y S}}{ }^{2 h}$ & $\mathbf{S D E P Y S}^{i}$ & $\boldsymbol{q}_{\mathbf{S A}^{2 j}}$ & $\mathbf{S D E P S A}^{k}$ \\
$\mathrm{~A}$ & 1.65 & 5 & 0.01 & 0.05 & 0.85 & 0.26 & 0.31 & -0.83 & 0.39 & 0.56 & 0.2 \\
$\mathrm{C}$ & 1.7 & 5 & 0.01 & 0.05 & 0.88 & 0.25 & 0.26 & -1.01 & 0.41 & 0.6 & 0.19 \\
$\mathrm{HD}$ & 1.1 & 3 & 0.009 & 0.045 & 0.83 & 0.29 & 0.25 & -0.57 & 0.37 & 0.49 & 0.21 \\
$\mathrm{OA}$ & 1.6 & 5 & 0.01 & 0.05 & 0.84 & 0.29 & 0.25 & -0.65 & 0.37 & 0.15 & 0.27 \\
$\mathrm{~N}$ & 1.6 & 5 & 0.01 & 0.05 & 0.88 & 0.36 & 0.24 & -0.93 & 0.4 & 0.15 & 0.27 \\
$\mathrm{NA}$ & 1.65 & 5 & 0.01 & 0.05 & 0.88 & 0.36 & 0.24 & -0.90 & 0.4 & 0.58 & 0.19 \\
\hline
\end{tabular}

${ }^{a}$ Spacing between the grid nodes. ${ }^{b}$ Cutoff of very low or very high energy value. ${ }^{c}$ Zeroing of very low data points. ${ }^{d}$ Minimum starndard deviation value. ${ }^{e}$ Coventional square-correlation coefficient. ${ }^{f} \mathrm{Cross}$ validation coefficient. ${ }^{g}$ Standard deviation error of prediction. ${ }^{h}$ Average Cross validation coefficient using leave-one-out (LOO) method obtained after Y-scrambling process, using 100 iterations. ${ }^{i}$ Standard deviation error of prediction obtained after Y-scrambling process using 100 iterations. ${ }^{j}$ Cross validation coefficient obtained after simulated annealing. ${ }^{k}$ Standard deviation error of prediction after simulated annealing. 
Table 2S. 2-D and 3-D Structures, Features and Their Values of the Selected Template Molecules

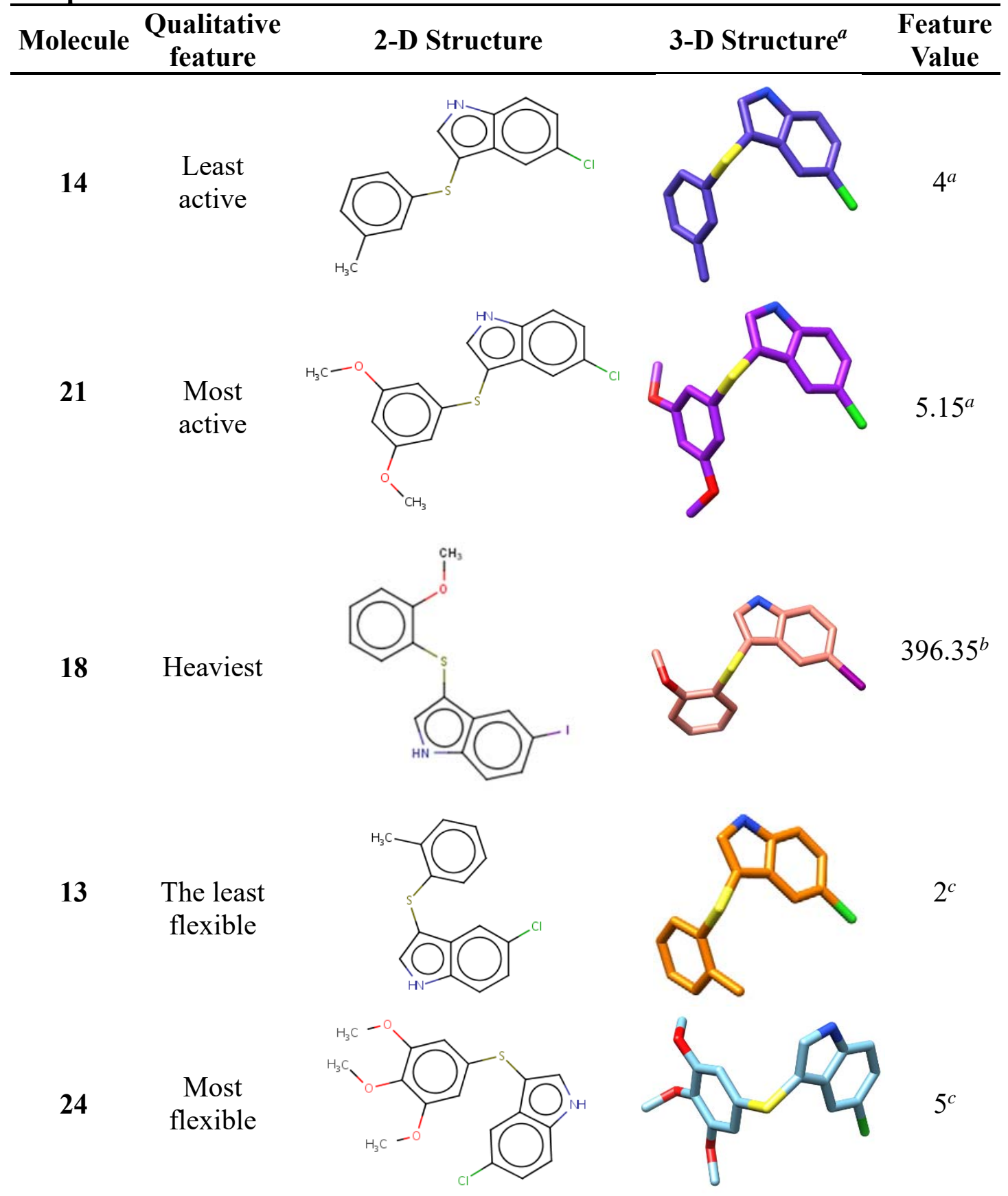

$\overline{{ }^{a} \text { Biological activity expressed in } \mathrm{pIC}_{50}\left(-\log \left[-\mathrm{IC}_{50(\mu \mathrm{M})} * 10^{-6}\right]\right) .{ }^{b} \text { Molecular mass. }{ }^{c} \text { Number of rotatable }}$ bonds. 
Table 3S. Grid Optimization and VPO Analysis Parameters

\begin{tabular}{cccc}
\hline Details & Min & Increment & Max \\
\hline $\begin{array}{c}\text { Performs models at different } \\
\text { grid spacing }\end{array}$ & $0.5(\ddot{\mathrm{A}})$ & $0.05(\ddot{\mathrm{A}})$ & $2(\ddot{\mathrm{A}})$ \\
$\begin{array}{c}\text { Maximum } \text { minimum energy }_{\text {cutoff value }}^{b} \\
\begin{array}{c}\text { Maximum zeroing } \\
\text { aximum }\end{array}\end{array}$ & 1 & 1 & 5 \\
Minimum MIF standard $_{\text {deviation }}{ }^{c}$ & 0.001 & 0.001 & 0.01 \\
\hline
\end{tabular}

${ }^{a}$ Spacing between the grid nodes. ${ }^{b}$ Cutoff of very low or very high energy value. ${ }^{c}$ Zeroing of very low data points. ${ }^{d}$ Minimum standard deviation value. 
Table 4S. AdmetSAR Data for Diverse Chemicals Associated with Known Absorption, Distribution, Metabolism, Excretion and Toxicity Profiles for Compounds 5 and 21

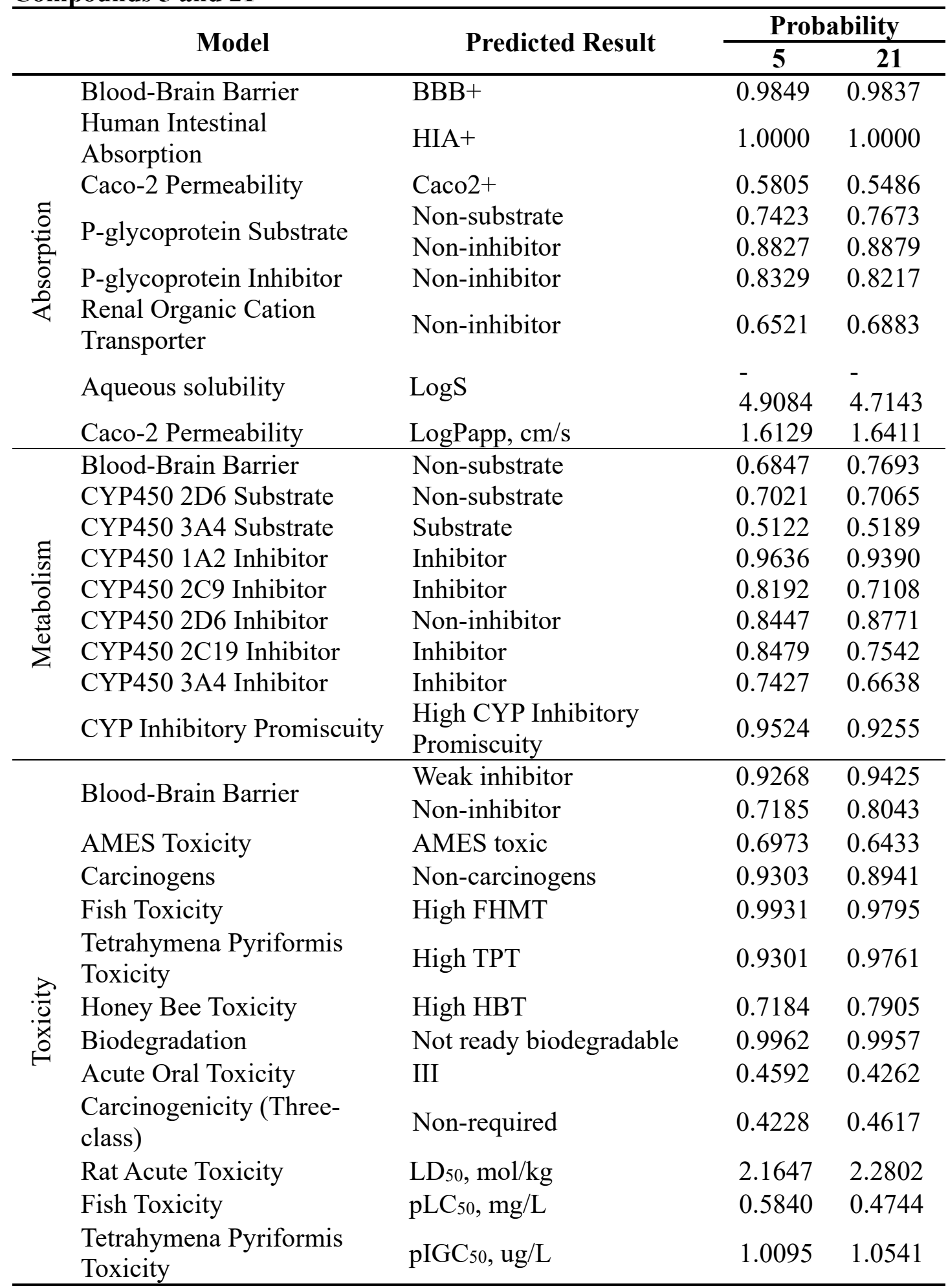


Table 5S. Calculated Drug Likeness Properties for Compounds 5 and 21 by Molinspiration Web Server

\begin{tabular}{cccc}
\hline & ADMET Features & $\mathbf{5}$ & $\mathbf{2 1}$ \\
\hline & miLogP $^{a}$ & 4.72 & 4.75 \\
& TPSA $^{b}$ & 25.02 & 34.26 \\
Natoms $^{c}$ & 19 & 21 \\
MW $^{d}$ & 289.79 & 319.81 \\
& nON $^{e}$ & 2 & 3 \\
& nOHNH $^{f}$ & 1 & 1 \\
& N violations $^{g}$ & 0 & 0 \\
Nrotb $^{h}$ & 3 & 4 \\
& Volume $^{i}$ & 241.64 & 267.19 \\
\hline
\end{tabular}

${ }^{a} \log \mathrm{P}$ (octanol/water partition coefficient) is calculated by the methodology developed by Molinspiration as a sum of fragment-based contributions and correction factors. Method is very robust and is able to process practically all organic, and most organometallic molecules. ${ }^{b}$ Molecular Polar Surface Area TPSA is calculated as a sum of fragment contributions. O- and N- centered polar fragments are considered. PSA has been shown to be a very good descriptor characterizing drug absorption, including intestinal absorption, bioavailability, Caco-2 permeability and blood-brain barrier penetration. ${ }^{~ N}$ umber of heavy atoms. ${ }^{d}$ Molecular Volume. Method for calculation of molecule volume developed at Molinspiration is based on group contributions. These have been obtained by fitting sum of fragment contributions to "real" 3D volume for a training set of about twelve thousand, mostly druglike molecules. 3D molecular geometries for a training set were fully optimized by the semiempirical AM1 method. ${ }^{e}$ Number of hydrogens atoms on oxygens and nitrogens. ${ }^{f}$ Number of oxygen and nitrogen atoms. ${ }^{g}$ Lipinsky rule of 5 properties is set of simple molecular descriptors used by Lipinski in formulating his "Rule of 5". The rule states, that most "drug-like" molecules have $\log \mathrm{P}<=5$, molecular weight $<=500$, number of hydrogen bond acceptors $<=10$, and number of hydrogen bond donors $<=$ 5. Molecules violating more than one of these rules may have problems with bioavailability. ${ }^{h}$ This simple topological parameter is a measure of molecular flexibility. It has been shown to be a very good descriptor of oral bioavailability of drugs. Rotatable bond is defined as any single non-ring bond, bounded to nonterminal heavy (i.e., non-hydrogen) atom. Amide C-N bonds are not considered because of their high rotational energy barrier. ${ }^{i}$ Molecular volume. 
Table 6S. Volsurf Calculated ADMET Properties for Compounds 5 and 21

\begin{tabular}{cccrcccc}
\hline Cpd & PC & $\mathbf{C P}^{\boldsymbol{a}}$ & \multicolumn{1}{c}{$\mathbf{P B}^{\boldsymbol{b}}$} & \multicolumn{1}{c}{ SKIN $^{\boldsymbol{c}}$} & SOLY $^{\boldsymbol{d}}$ & VD $^{\boldsymbol{e}}$ & MetStab $^{\boldsymbol{f}}$ \\
\hline \multirow{5}{*}{$\mathbf{5}$} & 1 & 1.74642 & 100.5550 & -1.21385 & -5.15336 & -0.828831 & 29.8349 \\
& 2 & 1.81253 & 92.4702 & -0.67126 & -5.04777 & -0.588632 & 37.1178 \\
& 3 & 1.66288 & 99.5783 & -1.24524 & -5.00167 & -0.764128 & - \\
& 4 & 1.63456 & 101.8850 & -1.17571 & -5.13061 & -0.806878 & - \\
& 5 & 1.61668 & 102.3430 & -0.86534 & -5.27352 & -0.801761 & - \\
\hline \multirow{2}{*}{$\mathbf{2 1}$} & 1 & 1.47761 & 97.2059 & -1.71994 & -4.97672 & -0.693771 & 32.0082 \\
& 2 & 1.56267 & 94.6379 & -1.09169 & -5.06103 & -0.485308 & 35.9610 \\
& 3 & 1.51795 & 104.2160 & -1.54913 & -5.2087 & -0.686635 & - \\
& 4 & 1.50326 & 111.0530 & -1.47366 & -5.27557 & -0.720919 & - \\
\hline
\end{tabular}

All values for all the computed principal components are reported. ${ }^{a} \mathrm{CACO} 2$ permeability computed via a PLS model derived by fitting VolSurf+ descriptors to experimental data on $\mathrm{CACO} 2$ cells permeability. The value is only qualitative (not quantitative). ${ }^{b}$ Protein binding computed via a PLS model derived by fitting VolSurf+ descriptors to experimental data of protein binding. ${ }^{~}$ Skin permeability computed via a PLS model derived by fitting VolSurf+ descriptors to experimental data on skin permeability. The value is quantitative $(\mathrm{cm} / \mathrm{h}) .{ }^{d}$ Intrinsic solubility computed via a PLS model derived by fitting VolSurf+ descriptors to the logarithm of experimental intrinsic solubility (mol/Litre at $25^{\circ} \mathrm{C}$ ), also called intrinsic solubility. ${ }^{e}$ Volume of distribution computed via a PLS model

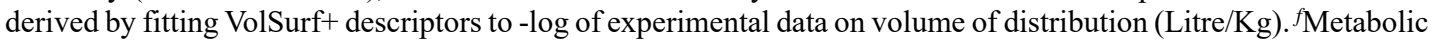
Stability as percentage of the remaining compound after incubation with human CYP3A4 enzyme. Values greater than 50 indicate stable behavior (100 is the maximum stability). Values lower than 50 are less precise and indicate metabolic instability. 